\title{
Design of an acrylic wastewater treatment method based on deep oxidation technology
}

\author{
Wei Liang ${ }^{1}$ \\ ${ }^{1}$ Acrylic fiber factory of Daqing Petrochemical Company in Heilongjiang Province, Daqing, China
}

\begin{abstract}
Acrylic fiber is one of the earliest developed chemical fiber species in China. Acrylic products are fluffy, warm, soft to the touch, and have good weather resistance and anti-mildew and anti-moth properties. Acrylic fiber has a wide range of applicability, but the wastewater generated during its production is a major problem facing the world. The complex composition and high toxicity of acrylic wastewater pollutants, the presence of many oligomers and other difficult to biodegrade, as well as the presence of sulfate, sulfite and other bioinhibitory components, make the biochemical properties of acrylic wastewater very low, making it very difficult to treat. In this paper, we propose an acrylic wastewater treatment method based on deep oxidation technology to solve this problem, which can effectively carry out wastewater treatment and make it meet the national discharge standards.
\end{abstract}

\section{Introduce}

In recent years, the demand for acrylics has been increasing, and the mass production of acrylonitrile has led to increasing pollution problems in its production. Both the production process and the storage and transportation of acrylonitrile inadvertently lead to its effluent, which can cause pollution of water resources and pose a threat to human health if not treated. Various data show that due to the physical and chemical properties of acrylonitrile, trace amounts of acrylonitrile dissolved in water bodies can cause high toxicity and potential "tritoxicity" to water bodies. The high toxicity of acrylonitrile has led to its appearance on the list of priority toxic chemicals and the blacklist of priority pollutants in water in China. In recent years, many scholars from different countries have studied the degradation of this highly toxic wastewater. For the treatment of acrylonitrile wastewater, the research focuses on new processes that are efficient, resourceful and harmless. ${ }^{[1]}$

\section{Common methods for biochemical treatment of acrylic wastewater}

For the treatment of acrylic production wastewater, the current research is mostly focused on the development of end-of-pipe treatment technology. Pre-treatment mainly focuses on how to improve the biochemical properties of wastewater and remove biologically inhibitory components; subsequent biochemical treatment mainly focuses on reducing the discharge of COD and ammonia nitrogen in the effluent, and also includes the combination of these methods and the study of the adopted process route. The main purpose of acrylic wastewater treatment is to remove difficult biodegradable substances, and the main treatment technologies are coagulation, internal electrolysis, air floatation, advanced oxidation, ultrasonic wave, membrane filtration, catalytic ozone method, microwave method, bentonite and lime treatment of acrylic wastewater, photocatalytic oxidation technology, Fenton oxidation technology and catalytic wet oxidation technology. ${ }^{[2]}$ The technologies studied in this paper are mainly those in coagulation and oxidation.

\subsection{Coagulation technology}

Coagulation is to add coagulant to the wastewater to make the colloid and suspended particles in the wastewater coalesce into larger flocs, so as to separate them from the water and achieve the purpose of purifying water quality. Coagulation includes two processes: coagulation and coagulation. Coagulation is the destabilization of colloidal particles coagulation into small flocs, coagulation is in the role of the catalyst will be small flocs coagulation into a large volume of coagulation body, so as to complete the entire coagulation process. Coagulation mechanism varies because of the coagulant and coagulation conditions. At present, the coagulation mechanism mainly includes: adsorption electro-neutralization, adsorption bridging, compression double layer, net trapping, etc. ${ }^{[3]}$ The action forces mainly include hydrogen bonding, polarization, and the coagulation of the coagulant. The main forces are hydrogen bonding, polar groups, covalent bonds, electrostatic gravitational force and van der Waals force, etc. Which force plays a deterministic role is determined by the structure of the polymer itself and the characteristics of the colloid.

Different flocculants have different flocculation effect in treating acrylic process wastewater. For acrylic

* Corresponding author: liangwei1980@126.com 
wastewater, inorganic polymer flocculants have better treatment effect than organic polymer flocculants, among which polymerized ferric sulfate (PFS) has the best flocculation effect; inorganic and organic compound flocculants have better coagulation effect than single inorganic and organic flocculants, and the best compound combination is PFS and cationic polyacrylamide, with a certain amount of After adding a certain amount of $\mathrm{MgSO} 4$, the modified polyacrylamide with $\mathrm{MgSO} 4$ and PFS can achieve a COD removal rate of $32.5 \%$.

\subsection{Fenton oxidation technology}

Fenton advanced oxidation technology is a chemical oxidation water treatment technology invented by H.J.H. Fenton more than 100 years ago, which does not require high temperature and pressure and simple process equipment. When degrading organic matter with water, the hydroxide radicals can eventually oxidize the organic matter to the simplest $\mathrm{H} 2 \mathrm{O}$ and $\mathrm{CO} 2$ molecules by initiating chain reactions. The reaction mechanism is shown in Figure 1.

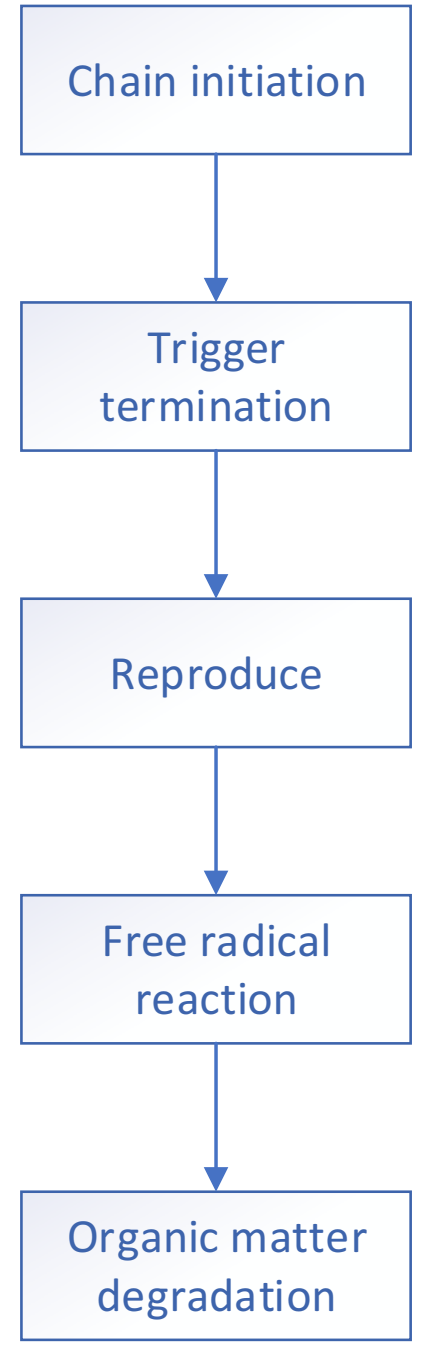

Fig. 1. Fenton oxidation technology process.

The organic degradation kinetic equation is:

$$
-\frac{\mathrm{d}[\mathrm{RH}]}{\mathrm{dt}}=\frac{\mathrm{K}_{1} \mathrm{~K}_{3}}{\mathrm{~K}_{2}} \cdot \frac{[\mathrm{RH}]\left[\mathrm{H}_{2} \mathrm{O}_{2}\right]}{\frac{\Delta\left[\mathrm{H}_{2} \mathrm{O}_{2}\right]}{2 \Delta\left[\mathrm{Fe}^{2+}\right]}}
$$

where $[\mathrm{RH}]$ is the concentration of organic matter. If
$\mathrm{K} 2>>\mathrm{K} 3$, under acidic conditions, because $\Delta[\mathrm{H} 2 \mathrm{O} 2] \approx$ $2 \Delta[\mathrm{Fe} 2+]$, the organic degradation kinetic equation is:

$$
-\frac{\mathrm{d}[\mathrm{RH}]}{\mathrm{dt}}=\frac{\mathrm{K}_{1} \mathrm{~K}_{3}}{\mathrm{~K}_{2}} \cdot[\mathrm{RH}]\left[\mathrm{H}_{2} \mathrm{O}_{2}\right]
$$

The amount of $\mathrm{H} 2 \mathrm{O} 2$ and $\mathrm{Fe} 2+$ salt input varies depending on the organic matter. fe2+ itself has flocculation effect and can remove COD from the effluent.

\subsection{Wet catalytic oxidation technology}

Catalytic wet oxidation technology is an advanced oxidation technology developed in the 1970s based on wet oxidation, using highly efficient and stable catalysts to reduce the activation energy of the reaction, thereby reducing the temperature and pressure required for the reaction and improving the treatment effect. Catalytic wet oxidation technology has been used industrially in Japan and other countries, but it has been studied later in China. According to the different states of catalysts, catalysts can be divided into two categories: homogeneous catalysts and non-homogeneous catalysts. The homogeneous catalyst, which was studied more in the early stage, is a soluble catalyst added to the reaction system to catalyze the reaction. It has good reaction performance, mild reaction and specific selectivity, and the current research focuses on non-homogeneous catalytic oxidation. The research of non-homogeneous catalytic wet oxidation focuses on efficient and stable multiphase catalysts. ${ }^{[4]}$

From the current research results, the more studied and most valued homogeneous catalytic oxidants are soluble transition metal salts, and the catalytic activity of copper is more obvious, and the addition of copper ions mainly triggers the free radical chain of oxidation reaction by forming intermediate complexation products after dehydrogenation. The reaction flow is shown in Figure 2.

There are many methods for the preparation of catalysts, and the following two methods were chosen for the preparation of copper-based catalysts in this design.

(1) Precipitation method: The configured $\mathrm{Cu}(\mathrm{NO} 3) 2$ solution was adjusted to $\mathrm{pH} 10$ with ammonia as precipitant, aged in a water bath shaker at a constant temperature of $80^{\circ} \mathrm{C}$ for $2 \mathrm{~h}$, then filtered and washed, and the filtered solid sample was dried in an oven and placed in a muffle furnace at $120^{\circ} \mathrm{C}$ for $1 \mathrm{~h}$. Then the temperature was slowly and uniformly increased to $500-550^{\circ} \mathrm{C}$ (the set roasting temperature), and the time was started.

(2) Impregnation method: $\gamma-\mathrm{A} 12 \mathrm{O} 3$ was chosen as the carrier of the catalyst. In order to remove impurities, the catalyst was impregnated with distilled water for 8 hours before use, and the impregnation process was continuously stirred to resolve as many magazines as possible. After the impregnation, the $\gamma$-A12O3 was cleaned with distilled water, and then placed in an oven at a temperature of $120^{\circ} \mathrm{C}$ and dried for $12 \mathrm{~h}$, ready for use. Weigh a certain amount of dried $\gamma$-A $12 \mathrm{O} 3$ particles into a triangular flask, add an excess of $\mathrm{Cu}(\mathrm{NO} 3) 2$ solution (impregnation solution), put it on an oscillator, and filter it after 8 hours of reaction. The filtered solid was dried and placed in a muffle furnace for $1 \mathrm{~h}$ at $120{ }^{\circ} \mathrm{C}$, then the temperature was slowly and uniformly increased to 500$550{ }^{\circ} \mathrm{C}$ and the time was started. The roasted catalyst was crushed, sieved and placed in a desiccator. ${ }^{[5]}$ 


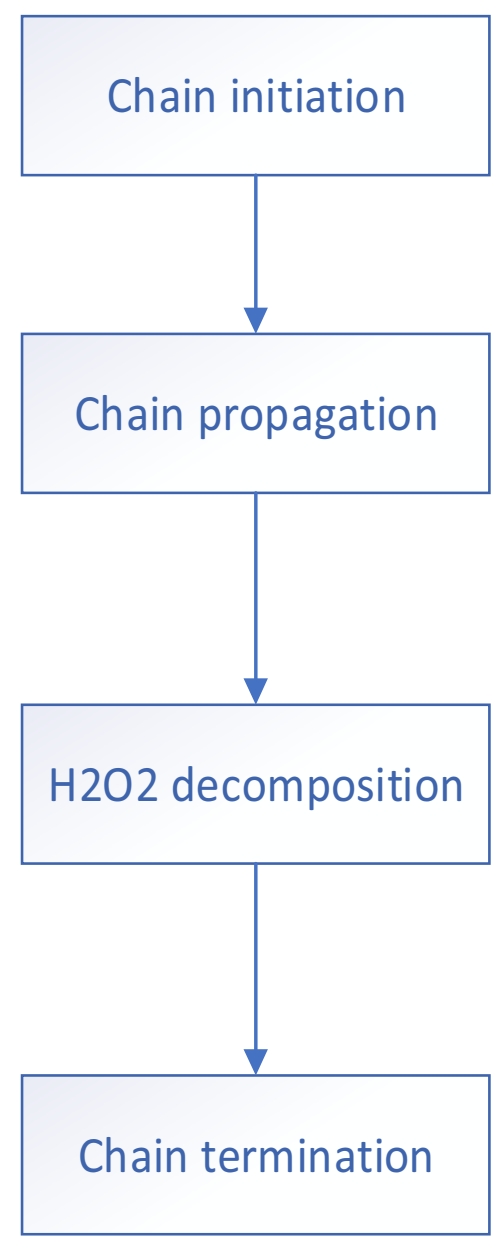

Fig. 2. Oxidation chain reaction flow.

\section{Design of acrylic wastewater treatment method}

\subsection{Kinetic model of catalytic wet oxidation reaction process}

The study of kinetics is an important part of the research on wet oxidation technology. By investigating the reaction kinetics, not only can we gain insight into the course of the reaction, understand the reaction mechanism and the control steps of the reaction, which can better promote the occurrence of the reaction, but also provide a basic theoretical basis for engineering design and process control through the establishment of mathematical models of kinetics. There are three main categories of kinetic models proposed so far: mechanistic, empirical and semiempirical models. The most commonly used is the empirical model, whose reaction rate equation is:

$$
-\frac{\mathrm{d}[\mathrm{c}]}{\mathrm{d}[\mathrm{t}]}=\mathrm{k}_{0} \operatorname{cxp}\left(-\mathrm{E}_{\mathrm{a}} / \mathrm{RT}\right)\left[\mathrm{c}_{1}\right]^{\mathrm{m}}\left[\mathrm{c}_{2}\right]^{\mathrm{n}}
$$

Where:

[c] - molar concentration of reactants, $\mathrm{mol} / \mathrm{L}$ or $\mathrm{mg} / \mathrm{L}$; $\mathrm{k} 0$ - refers to the prefactor, its value is related to $\mathrm{m}, \mathrm{n}$;

$\mathrm{Ea}$ - reaction $\mathrm{Ea}$ - activation energy of the reaction, $\mathrm{kJ} / \mathrm{mol}$;

$\mathrm{R}$ - gas constant. $8.314 \mathrm{~J} /(\mathrm{mol}-\mathrm{K})$;

c1 - concentration of organic matter, which can be expressed in terms of specific organic matter concentration or comprehensive index of water quality (e.g. COD);

c2 --Oxidant concentration, can be expressed as liquidphase DO or gas-phase oxygen partial pressure; $m$-reaction level of organic matter;

$\mathrm{n}$--reaction index of oxidant;

$\mathrm{t}$ - reaction time, min or $\mathrm{s}$;

$\mathrm{T}$ - reaction temperature, $\mathrm{K}$.

The main investigation of this design is the effect of catalytic wet oxidation on the comprehensive index COD of water quality. Therefore, the kinetic model of wet catalytic oxidation of acrylic wastewater is as follows:

$$
-\frac{\mathrm{d}[\mathrm{c}]}{\mathrm{dt}}=\mathrm{k}_{0} \exp \left(-\mathrm{E}_{\mathrm{a}} / \mathrm{RT}\right)[\mathrm{COD}]^{m}\left[\mathrm{O}_{2 \mathrm{ads}}\right]^{n}
$$

\subsection{Process design of treatment method}

In view of the problems of the existing process and technology, the acrylic wastewater treatment method is formulated by combining the actual site and the characteristics of wastewater quality. Firstly, the chemical method is used to pretreat the hard-to-degrade acrylic wastewater, and then the deep oxidation method is used to treat it, so as to ensure the removal of COD fundamentally.

In the design process, the influencing factors of coagulation and Fenton oxidation were analyzed using the single-factor method to determine the best parameter conditions for treating acrylic wastewater. The influence of wet catalyst preparation on the catalytic efficiency was studied, and the single-factor method was used to analyze the influence of pressure and temperature on the treatment effect of wet catalytic oxidation and to obtain the optimal reaction conditions. The pretreatment and catalytic wet oxidation were combined to treat acrylic wastewater to determine the optimal process. The reaction kinetics of catalytic wet oxidation process was initially explored, the corresponding kinetic model was established, and the model parameters and activation energy were investigated.

The proposed design is shown in Figure 3. 


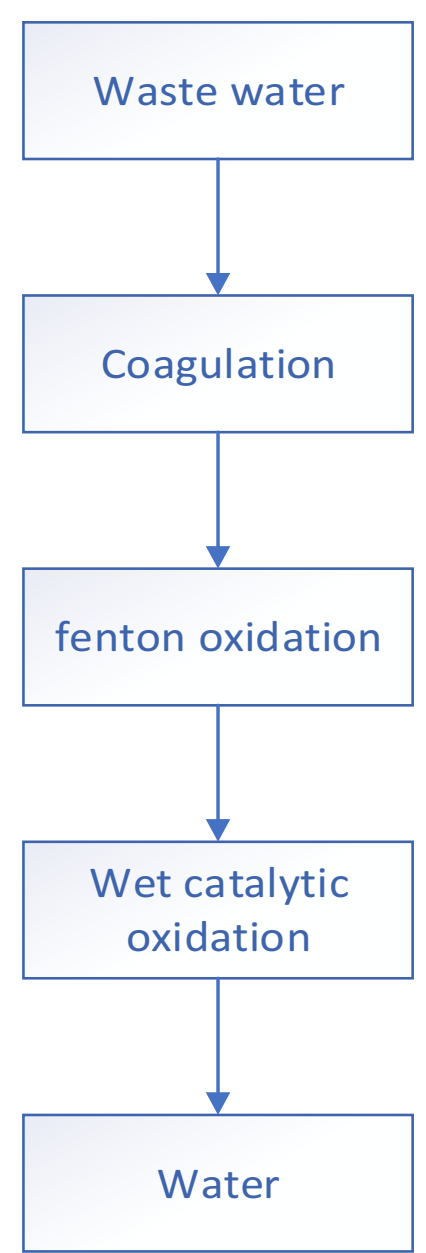

Fig.3. Wastewater treatment process

The experimental steps of each step treatment method are as follows.

(1) Firstly, the coagulation test was carried out by taking $200 \mathrm{~mL}$ of wastewater, adding polyaluminium and polyacrylamide at concentrations of $600 \mathrm{mg} / \mathrm{L}$ and $2 \mathrm{mg} / \mathrm{L}$ respectively under the condition of room temperature and $\mathrm{p} \mathrm{H}=8$, stirring at $200 \mathrm{r} / \mathrm{min}$ for $1 \mathrm{~min}$ and then stirring at $60 \mathrm{r} / \mathrm{min}$ for $15 \mathrm{~min}$ to make the reaction adequate, and taking the supernatant after standing for $30 \mathrm{~min}$, and taking a small amount to measure the COD concentration.

(2) Take $50 \mathrm{~mL}$ of supernatant for Fenton oxidation treatment. Add 30\% hydrogen peroxide (H2O2) solution $0.6 \mathrm{~mL}$, then add FeSO4 solution $4.2 \mathrm{~mL}$, adjust the $\mathrm{pH}$ to 3.5 , avoid light reaction for $120 \mathrm{~min}$, adjust the $\mathrm{pH}$ to 8 with sodium hydroxide respectively, terminate the oxidation reaction, stand for $30 \mathrm{~min}$, extract the supernatant, take a small amount to measure the COD concentration.

(3) The supernatant obtained from Fenton oxidation treatment was subjected to wet catalytic oxidation treatment. At a temperature of $250{ }^{\circ} \mathrm{C}$ and a partial pressure of oxygen of $2 \mathrm{MPa}, 200 \mathrm{mg} / \mathrm{L}$ of catalyst prepared by impregnation was added, and the reaction time was $90 \mathrm{~min}$, and the COD concentration was measured by condensing a sample.

The test results show that the combined coagulation + Fenton oxidation + wet catalytic oxidation process has high stability, and the removal rate of COD in the wastewater by this combined process reaches more than
$90 \%$, and the COD value of the treated effluent is less than $60 \mathrm{mg} / \mathrm{L}$.

\section{Conclusion}

In this paper, we firstly studied the pretreatment process of acrylic wastewater, using a combination of coagulation and Fenton advanced oxidation to pretreat acrylic wastewater, and then focused on the preparation and influencing factors of catalysts for catalytic wet oxidation treatment of acrylic wastewater, and finally conducted a preliminary investigation on the reaction kinetics of copper-based catalysts for catalytic wet oxidation treatment of acrylic wastewater. Through the experimental study on the actual acrylic wastewater, the process technology scheme of this experiment was determined as: coagulation-Fenton oxidation-wet catalytic oxidation. After this process to treat acrylic wastewater with wet oxidation method alone, the catalyst was prepared by precipitation method and impregnation method, and the experiments showed that the catalyst prepared by impregnation method had better catalytic effect, so the catalyst was prepared by impregnation method. It can effectively degrade the oligomers in acrylic wastewater, and the COD concentration of treated wastewater can meet the discharge requirements. In summary, the deep oxidation wastewater treatment process proposed in this study is effective in treating acrylic wastewater, and the wastewater effluent can meet the national standard, which has explored a feasible path for the treatment of actual acrylic wastewater, and its economic and technical properties can meet the requirements of wastewater treatment, which provides a certain research basis for future industrialization research and has strong practicality.

\section{References}

1. L. W. Liang,L. Y. Liu,F. Yang. Research on pretreatment technology of acrylic industrial wastewater $[\mathrm{J}]$. Advances in fine petrochemicals, 2019,20(04):26-28.

2. Zhang Yulong,Shen Yan,Lv Zhengping,Xu Yejun. Study of A/O-MBR process treatment based on acrylic plant wastewater[J]. Contemporary Chemical Industry,2019,48(07):1471-1475.

3. $\mathrm{Xu} \mathrm{K}$. Analysis of acrylonitrile wastewater treatment technology[J]. Contemporary chemical research,2018(08):59-60.

4. Wang J.E.,Wu J.J.,Yu J.Q.,Lei T.S. Study of wet catalytic hydrogen peroxide oxidation for the treatment of acrylic wastewater[J]. Contemporary Chemical Industry,2017,46(05):838-840+845.

5. Bai Fan. Research on pretreatment of acrylic wastewater by sponge iron type "iron-carbon microelectrolysis coupled with Fenton reagent" method[D]. Lanzhou Jiaotong University,2017. 